\title{
E2 Ubiquitin-Conjugating Enzyme
}

National Cancer Institute

\section{Source}

National Cancer Institute. E2 Ubiquitin-Conjugating Enzyme. NCI Thesaurus. Code C133711.

An aminoacyltransferase that catalyzes the second step in the ubiquitination reaction via the transfer of activated ubiquitin from an E1 ubiquitin-activating enzyme to itself via transthioesterification. 